\title{
Association of Long Sleep and Physical Inactivity with Incident Disability in Community-Dwelling Older Adults: A 5-Year Longitudinal Study
}

Sho Nakakubo ( $\nabla$ sho-n@ncgg.go.jp )

National Center for Geriatrics and Gerontology

Takehiko Doi

National Center for Geriatrics and Gerontology

Kota Tsutsumimoto

National Center for Geriatrics and Gerontology

Satoshi Kurita

National Center for Geriatrics and Gerontology

Hideaki Ishii

International University of Health and Welfare

Yuto Kiuchi

National Center for Geriatrics and Gerontology

Hiroyuki Shimada

National Center for Geriatrics and Gerontology

\section{Research Article}

Keywords: elderly, physical inactivity, long sleep duration, incident disability

Posted Date: October 11th, 2021

DOl: https://doi.org/10.21203/rs.3.rs-957328/v1

License: (9) (i) This work is licensed under a Creative Commons Attribution 4.0 International License.

Read Full License 


\section{Abstract \\ Background}

Good sleep durations and physical activity are essential factor for maintaining good health. This study examined whether sleep duration and physical inactivity were associated with incident disability among community-dwelling older adults.

\section{Methods}

A total of 4,372 adults aged $\geq 70$ years participated in a baseline assessment. We also assessed selfreported sleep duration and physical activity using the International Physical Activity Questionnaire Short Form at baseline assessment. We measured monthly incident disability, defined as the onset of being certified for personal support or care as required by Japanese public long-term care insurance over 5 years. The Cox proportional hazard regression analysis estimated hazard ratios (HRs) and 95\% confidence intervals (Cls) for incident disability according to long sleep duration ( $\geq 9 \mathrm{~h}$ ) and physical inactivity.

\section{Results}

Long sleep duration (HR 1.32, Cl 95\% 1.05-1.67) and physical inactivity (HR 1.33, Cl 95\% 1.13-1.57) were associated with incident disability in multivariate analyses. These results were also sustained in the model where both long sleep duration and physical inactivity were simultaneously submitted as independent variables. Furthermore, coexistence of long sleep duration and physical inactivity had higher risk of incident disability than each factor individually (HR 1.69, Cl 95\% 1.11-2.58), even though the synergistic interaction was mildly effective.

\section{Conclusions}

This study revealed that long sleep duration and physical inactivity were independently associated with the risk of incident disability among older adults. More attention should be paid to both sleep duration and physical activity in order to prevent incident disability.

\section{Introduction}

Disability in older adults, which is a growing public health concern, is associated with poor physical and mental health, increasing use of medical care, and high rate of institutionalization.[1,2] Identifying risk factors for incident disability is required to develop preventive strategies. Physical activity plays an important role in improving health outcomes, including risk of disability. A previous systematic review suggested that higher levels of physical activity were beneficial in preventing incident disability.[3] 
Good sleep condition in late life is an essential factor for maintaining good health. Poor sleep condition is associated with various adverse health outcomes, including disability in older adults.[4] Previous studies have identified insulin resistance,[5] autonomic nervous system dysregulation,[5, 6] metabolic derangement[7] and inflammation[8] as potential mediators between sleep disturbances and incident disability. Among sleep conditions (e.g., short/long sleep duration, excessive daytime sleepiness and insomnia), there has been growing evidence that the association of long sleep duration with health problems, mortality [9-13] and poor self-reported health.[14] Moreover, our previous study, covering a period of two years, showed that long sleep duration was associated with incident disability.[15]

However, when considering the association between sleep duration and health problems, it was inevitable to consider the association between sleep duration and physical activity, since it has been suggested that these two factors are related to each other. Long sleep duration was associated with a sedentary lifestyle and low levels of daytime physical activity,[16-18] while moderate physical activity was shown to have a positive impact on sleep quality among older adults in a systematic review.[19] Our previous study that revealed a longitudinal association of long sleep duration with incident disability was completed with only physical activity as a covariate.[15] Thus, in order to elucidate the association between long sleep duration and incident disability, an analysis that takes into account sleep duration and physical activity patterns should be conducted.

The primary aim of this study is thus to examine whether long sleep duration and physical inactivity were associated with incident disability among community-dwelling older adults. Second, we hypothesized that those who have both long sleep durations and physical inactivity show more risk of incident disability than those with either long sleep duration or physical inactivity only. In this study, an additive interaction between long sleep duration and low physical activity on incident disability was also examined.

\section{Methods}

\section{Setting and participants}

We assessed 5,257 individuals who were enrolled in part of the National Center for Geriatrics and Gerontology - Study of Geriatric Syndromes (NCGG-SGS) [20]. Inclusion criteria required that the participants were aged 70 years or older, lived in Nagoya City (from July 2013 to December 2013) at the time of examination. Four hundred participants were excluded from this study, based on the following criteria: 1$)$ history of stroke, Parkinson's disease or Alzheimer's disease $(n=360) ; 2)$ already certified by long-term care insurance $(\mathrm{LTCl})$ at any level during the assessment $(n=17) ; 3)$ inability to perform basic daily living tasks such as eating, grooming, bathing and using stairs $(n=6) ; 4)$ severe cognitive impairment [Mini-Mental State Examination (MMSE) [21] < $20(n=146)] ; 5)$ a missing value in our assessment $(n=168)$. Moreover, those who moved elsewhere $(n=35)$ or died $(n=123)$ during the follow-up period were also excluded. The final sample consisted of 4,372 people (mean age 75.9 \pm 4.2 years, 2,343 
women). Informed consent was obtained from all participants before their inclusion in the study, and the Ethics Committee of the National Center for Gerontology and Geriatrics approved the study protocol.

\section{Measures}

\section{Certification of need for care in the LTCI system}

The nationally uniform criteria for long-term care need certification were objectively established by the Japanese government, and certification of need with respect to levels of care for older adults is determined based on evaluation results by the Certification Committee for Long-Term Care Need in municipalities in accordance with these basic guidelines. Participants were followed monthly for incident certification of care needs according to the LTCI system during the 5 years after the baseline assessment. We defined incident disability as the point at which a participant was certified as needing care or support according to LTCl classification. The computer-aided standardized needs-assessment system used by the mandatory social LTCl system categorizes people into 7 levels of needs. [22]

\section{Sleep duration and physical activity}

Sleep duration and physical activity were assessed by self-reporting. We asked participants about their usual sleep and wake times, and the difference between them were calculated as their sleep duration. According to their self-reported sleep duration, the participants with sleep duration of $\geq 9 \mathrm{~h}$ were assigned to long sleep duration [15].

Physical activity was measured using the International Physical Activity Questionnaire - Short Form (IPAQ-SF).[23] The IPAQ-SF assesses usual 7 days' physical activity for each item (i.e., walking, moderate physical activity and vigorous physical activity) to estimate the time spent engaged in 10 or more minutes of physical activity per usual week. Participants were required to report the frequency and duration of their engagement in each item of physical activity. According to the official IPAQ guidelines, walking, moderate physical activity and vigorous physical activity were respectively assigned an intensity of 3.3, 4.0, and 8.0 METs, and total weekly physical activity (MET-min week-1) was calculated by adding the products of reported time for each item by a MET value that was specific to each category of physical activity. Both the total volume and the number of day/sessions are included in the IPAQ analysis algorithms. Participants were identified by three levels of physical activity - low, moderate and high referring to the official IPAQ guidelines[24] and were divided into two groups in this study: inactive (low) or active (moderate or high) groups.

\section{Sociodemographic variables and covariates}

Data on sociodemographic variables including sex, age and educational level (years) were collected, along with medical history, weight $(\mathrm{kg})$ and height $(\mathrm{m})$. Body mass index (BMI) was derived as weight in kilograms divided by the square of height in meters. Participants were asked about medical diagnoses (heart disease, diabetes, respiratory disease, hypertension and hyperlipidemia) and medications in faceto-face interviews. Depressive symptoms were measured using the Geriatric Depression Scale (range 0- 
15),[25] with higher scores indicating more depressive symptoms. MMSE was used as a screening tool for global cognitive function (range $0-30$, with higher scores indicating better functioning).[21] For smoking and alcohol drinking status, participants were categorized as current, past, or never smokers or alcohol drinkers, respectively.

\section{Statistical analysis}

All analyses were performed using SPSS 25.0 for Windows (SPSS Japan Inc., Tokyo, Japan) and statistical significance was set at $p<0.05$. The characteristics are summarized as mean \pm standard deviation (SD) for continuous variables and as count and percentage for categorical variables. Comparisons between independent and incident disability groups were performed using $t$-test and Pearson's chi-squared test for categorical data. Univariate and multivariate Cox proportional hazard regression analyses were conducted to assess hazard ratios (HR) with $95 \%$ confidence intervals (Cl) of long sleep duration and low physical activity for the risk of incident disability. In multivariate analysis, potential confounders included age, sex, BMl, education level, number of medications, MMSE score, medical history, depressive symptoms, smoking habits and alcohol consumption. Sleep duration and physical activity were individually (Model 1 and Model 2) and simultaneously (Model 3) submitted as independent variables.

Furthermore, the participants were also classified according to the combination of sleep duration and physical activity, as follows: 1) not long sleep duration \& active (Not long \& Active); 2) not long sleep duration \& inactive (Not long \& Inactive); 3) long sleep duration \& active (Long \& Active); 4) long sleep duration \& inactive (Long \& Inactive). Kaplan-Meier survival analysis for the incident disability was performed to compare 4 groups. Univariate and multivariate Cox proportional hazard regression analyses were also conducted with the same covariates. In addition, to assess an additive interaction between long sleep duration and low physical activity, we estimated the relative excess risk due to interaction (RERI) using the method described by Rothman.[26, 27] RERI between two factors (A and $B$ ) is defined as 'departure from additive effects' and is calculated as follows using adjusted HRs:[28]

$R E R I=H R(A \& B)-H R(A)-H R(B)+1$

RERI $<0, R E R I=0$ and RERI $>0$ indicate antagonistic interaction, absence of interaction and synergistic interaction, respectively.

\section{Results}

Of the 4,372 participants initially included, 878 participants $(20.1 \%)$ developed a disability during the 5year follow-up period. The differences in characteristics between independent and incident disability groups in the first wave are summarized in Table 1. The number of participants with long sleep duration was $267(6.1 \%)$ and that of participants with low physical activity was $713(16.3 \%)$.

In the crude model, Cox proportional hazard regression showed that the long sleep duration (HR: $1.72, \mathrm{Cl}$ 95\%: 1.37-2.16) and low physical activity (HR: 1.60, Cl 95\%: 1.37-1.88) had a higher rate of incident 
disability. These results were also sustained in multivariate analyses adjusted for covariates (Table 2, Model 1 and Model 2). Furthermore, both long sleep duration and physical inactivity were included in the multivariate Cox proportional hazard regression analysis as explanatory variables using the forced entry method adjusted for the other covariates. The results showed that long sleep duration and physical inactivity independently had an impact on incident disability (Table 2, Model 3).

Kaplan-Meier survival curves showed the highest rate of incident disability in the long sleep duration \& low physical activity group (Fig. 1, $p<0.001$ ). Compared with the Not long \& Active group, other groups showed a significantly higher rate of incident disability $(p<0.01$ for all). Long \& Inactive showed a significantly higher rate of incident disability than Not long \& Inactive $(p=0.007)$ and Long \& Active $(p=$ $0.029)$. There was no significant difference in the rate of incident disability between Not long \& Inactive and Long \& Active $(p=0.690)$.

In the Cox proportional hazard regression analysis, a crude model indicated that Not long \& Inactive (HR: 1.58, Cl 95\%: 1.33-1.86), Long \& Active (HR: 1.66, Cl 95\%: 1.27-2.17) and Long \& Inactive (HR: 2.82, Cl 95\%: 1.86-4.28) had significant association with incident disability, compared with the Not long \& Active group. Multivariate analysis adjusted for covariates showed similar association (Fig. 2). The RERI was 0.04 between long sleep duration and low physical activity with respect to incident disability, indicating a slight synergistic interaction.

\section{Discussion}

Our study revealed that long sleep duration and physical inactivity were independently associated with the risk of incident disability among older adults. These results were sustained after adjusted for age, sex, $\mathrm{BMI}$, education, medication, medical history, current drinking habit, current smoking habit, GDS score and MMSE score. Coexistence of long sleep duration and physical inactivity had a higher risk of incident disability than each factor individually, even though the synergistic interaction was mildly effective.

In this study, long sleep duration was shown to be associated with incident disability during a 5-year follow-up period. This result is consistent with our previous study conducted among other participants during a 2-year follow-up period.[15] Other previous cross-sectional studies showed that long sleep duration was also associated with other health adverse outcomes, such as physical frailty[29] and social frailty.[30] Additionally, long sleep duration was a risk factor for cognitive decline after 4 years among older adults.[31] These functional declines are included in geriatric syndromes and are serious risk factors for incident disability.[32-34] Although our study could not examine the cause of incident disability in detail, these functional declines would be mediated by the association between long sleep duration and incident disability. Furthermore, long sleepers were also found to have an increased wake after sleep onset (WASO) frequency and lower sleep efficiency.[35] Longitudinal study revealed that sleep complaints and lower sleep efficiency were associated with incident ADL disability among older adults.[4] [36] Therefore, the poor sleep quality would also be an underlying factor behind the association between long sleep duration and incident disability. 
Physical inactivity was also independently associated with incident disability in this study. In older adults, physical activity contributes to the prevention of disability caused by chronic diseases or functional decline.[37-39] Physical inactivity was associated with incident disability over a 9-year period among older adults.[40] Our result was in line with these previous studies. IPAQ-SF, which was used to assess physical activity in our study and allows us to assess physical activity and classify it according to three levels, can be easily used in clinical or research settings with lower respondent burden. Previous studies reported that physical activity assessed by IPAQ-SF has a cross-sectional association with health-related outcomes including quality of life, metabolic syndrome, sarcopenia and mental health.[41-44] These studies would support the association between IPAQ-assessed physical inactivity and incident disability.

Coexistence of long sleep duration and physical inactivity showed a higher hazard ratio of incident disability than each factor individually. However, RERI between long sleep duration and low physical activity with respect to incident disability was small, indicating that the synergistic interaction was mildly effective. The effects of long sleep duration and physical inactivity on incident disability would be not synergistic but independent, although several studies have observed that long sleep duration is associated with a sedentary lifestyle and low levels of daytime physical activity.[16-18] Further studies would be needed to examine the association of all-day activity, including daytime sleep or other activities, with incident disability.

A major strength of this study was its use of a prospective cohort design, which can address the causality between sleep duration and incident disability among older adults using large cohort data. However, this study had several limitations. First, self-reported measures of sleep duration were utilized, rendering the study incapable of performing a truly objective assessment of sleep characteristics, such as an assessment performed using actigraphy. Previous research suggested that self-reported long sleepers, [45] as well as average sleepers,[46] tend to overestimate their total sleep time, instead considering their entire time in bed. Similarly, self-reports of physical activity are likely to overestimate time spent in all intensities of physical activity.[47] Further studies that assess sleep duration and physical activity objectively using devices might show different results from ours, as we used self-report questionnaires to assess sleep duration and physical activity. Second, there were other potential confounders that we could not assess, such as drug therapy. Third, medical diagnoses were obtained by self-report, not by consulting medical records. Furthermore, given the absence of random sampling methods, our participants were exclusively those who had the ability to receive health checkups from their homes. Therefore, our results may not be directly applicable to all older adults in Japan, which is one of the limitations of this study.

\section{Conclusion}

We revealed that long sleep duration and physical inactivity were independently associated with the risk of incident disability among older adults from longitudinal cohort data during a 5-year follow-up period. These results suggest that more attention should be paid to both sleep duration and physical activity in order to prevent incident disability among older adults. Further research is needed to examine the 
association of long sleep duration and physical inactivity with incident disability using objective assessment methods.

\section{Declarations}

\section{Ethics approval and consent to participate}

Informed consent was obtained from all participants before their inclusion in the study. The Ethics Committee of the National Center for Gerontology and Geriatrics approved the study protocol and all methods were carried out in accordance with relevant guidelines and regulations.

\section{Consent for publication}

Not applicable.

\section{Availability of data and materials}

All data generated or analysed during this study are included in this published article.

\section{Competing interests}

The authors declare no competing conflicts of interest.

\section{Funding}

This work was supported by Health and Labour Sciences Research Grants (Comprehensive Research on Aging and Health); the Strategic Basic Research Programs (RISTEX Redesigning Communities for Aged Society), Japan Science and Technology Agency; Research project on health and welfare promotion for the elderly; and Research Funding for Longevity Sciences $(24-18,25-26)$ from the National Center for Geriatrics and Gerontology, Japan. The authors also received financial support through a Grant-in-Aid for Scientific Research (C) (18K11122, 21K11616).

\section{Authors' contributions}

SN designed and supervised all aspects of the study implementation and drafted the manuscript. TD and HS contributed to the conception and design of the study and provided feedback on the study implementation. KT contributed to the design of the study, and subject recruitment and screening. SK, IH, YK contributed to subject recruitment and screening. All authors participated in drafting the manuscript and revising it critically for important intellectual content. All authors have read and approved the manuscript.

\section{Acknowledgements}

We thank the Midori Ward Office for providing help with participant recruitment. 


\section{References}

1. Fried LP, Guralnik JM: Disability in older adults: Evidence regarding significance, etiology, and risk. $J$ Am Geriatr Soc 1997, 45(1):92-100.

2. Fried LP, Ferrucci L, Darer J, Williamson JD, Anderson G: Untangling the concepts of disability, frailty, and comorbidity: Implications for improved targeting and care. J Gerontol a-Bio/ 2004, 59(3):255263.

3. Paterson DH, Warburton DE: Physical activity and functional limitations in older adults: a systematic review related to Canada's Physical Activity Guidelines. The international journal of behavioral nutrition and physical activity 2010, 7:38.

4. Park M, Buchman AS, Lim AS, Leurgans SE, Bennett DA: Sleep complaints and incident disability in a community-based cohort study of older persons. The American journal of geriatric psychiatry : official journal of the American Association for Geriatric Psychiatry 2014, 22(7):718-726.

5. Spiegel K, Leproult R, Van Cauter E: Impact of sleep debt on metabolic and endocrine function. Lancet 1999, 354(9188):1435-1439.

6. Meerlo P, Sgoifo A, Suchecki D: Restricted and disrupted sleep: effects on autonomic function, neuroendocrine stress systems and stress responsivity. Sleep Med Rev 2008, 12(3):197-210.

7. Schmid SM, Hallschmid M, Jauch-Chara K, Bandorf N, Born J, Schultes B: Sleep loss alters basal metabolic hormone secretion and modulates the dynamic counterregulatory response to hypoglycemia. J Clin Endocrinol Metab 2007, 92(8):3044-3051.

8. Irwin MR, Wang M, Campomayor CO, Collado-Hidalgo A, Cole S: Sleep deprivation and activation of morning levels of cellular and genomic markers of inflammation. Arch Intern Med 2006, 166(16):1756-1762.

9. Lee JSW, Auyeung TW, Leung J, Chan DK, Kwok T, Woo J, Wing YK: Long Sleep Duration Is Associated With Higher Mortality in Older People Independent of Frailty: A 5-Year Cohort Study. Journal of the American Medical Directors Association 2014, 15(9):649-654.

10. Lee WJ, Peng LN, Liang CK, Chiou ST, Chen LK: Long sleep duration, independent of frailty and chronic Inflammation, was associated with higher mortality: A national population-based study. Geriatr Gerontol Int 2016.

11. Mesas AE, Lopez-Garcia E, Leon-Munoz LM, Guallar-Castillon P, Rodriguez-Artalejo F: Sleep duration and mortality according to health status in older adults. J Am Geriatr Soc 2010, 58(10):1870-1877.

12. Cappuccio FP, D'Elia L, Strazzullo P, Miller MA: Sleep duration and all-cause mortality: a systematic review and meta-analysis of prospective studies. Sleep 2010, 33(5):585-592.

13. Yeo Y, Ma SH, Park SK, Chang SH, Shin HR, Kang D, Yoo KY: A prospective cohort study on the relationship of sleep duration with all-cause and disease-specific mortality in the Korean Multi-center Cancer Cohort study. J Prev Med Public Health 2013, 46(5):271-281.

14. Stone KL, Ewing SK, Ancoli-Israel S, Ensrud KE, Redline S, Bauer DC, Cauley JA, Hillier TA, Cummings SR: Self-reported sleep and nap habits and risk of mortality in a large cohort of older women. J Am 
Geriatr Soc 2009, 57(4):604-611.

15. Nakakubo S, Doi T, Makizako H, Tsutsumimoto K, Hotta R, Ono R, Suzuki T, Shimada H: Sleep Duration and Excessive Daytime Sleepiness Are Associated With Incidence of Disability in Community-Dwelling Older Adults. Journal of the American Medical Directors Association 2016, 17(8):768 e761-765.

16. Kronholm E, Harma M, Hublin C, Aro AR, Partonen T: Self-reported sleep duration in Finnish general population. J Sleep Res 2006, 15(3):276-290.

17. Basner M, Fomberstein KM, Razavi FM, Banks S, William JH, Rosa RR, Dinges DF: American time use survey: Sleep time and its relationship to waking activities. Sleep 2007, 30(9):1085-1095.

18. Morgan $\mathrm{K}$ : Long sleep duration and all cause mortality in later life: A possible consequence of sedentary death syndrome. Sleep 2007, 30:A115-A115.

19. Vanderlinden J, Boen F, van Uffelen JGZ: Effects of physical activity programs on sleep outcomes in older adults: a systematic review. The international journal of behavioral nutrition and physical activity 2020, 17(1):11.

20. Shimada H, Makizako H, Lee S, Doi T, Lee S, Tsutsumimoto K, Harada K, Hotta R, Bae S, Nakakubo S et al: Impact of Cognitive Frailty on Daily Activities in Older Persons. J Nutr Health Aging 2016, 20(7):729-735.

21. Folstein MF, Robins LN, Helzer JE: THE MINI-MENTAL STATE EXAMINATION. Archives of General Psychiatry 1983, 40(7):812.

22. Tsutsui T, Muramatsu N: Care-needs certification in the long-term care insurance system of Japan. $J$ Am Geriatr Soc 2005, 53(3):522-527.

23. Craig CL, Marshall AL, Sjostrom M, Bauman AE, Booth ML, Ainsworth BE, Pratt M, Ekelund U, Yngve A, Sallis JF et al: International physical activity questionnaire: 12-country reliability and validity. Med Sci Sports Exerc 2003, 35(8):1381-1395.

24. International Physical Activity Questionnaire Web site [Internet]. Guidelines for data processing and analysis of the International Physical Activity Questionnaire (IPAQ)-Short and long Forms 2005. Available from: [http://www.ipaq.ki.se.]

25. Yesavage JA, Brink TL, Rose TL, Lum O, Huang V, Adey M, Leirer VO: Development and validation of a geriatric depression screening scale: a preliminary report. J Psychiatr Res 1982, 17(1):37-49.

26. KJ R: Epidemiology: an introduction.; 2002.

27. Andersson T, Alfredsson L, Kallberg H, Zdravkovic S, Ahlbom A: Calculating measures of biological interaction. Eur J Epidemiol 2005, 20(7):575-579.

28. Li R, Chambless L: Test for additive interaction in proportional hazards models. Ann Epidemio/2007, 17(3):227-236.

29. Nakakubo S, Makizako H, Doi T, Tsutsumimoto K, Hotta R, Lee S, Lee S, Bae S, Makino K, Suzuki T et al: Long and Short Sleep Duration and Physical Frailty in Community-Dwelling Older Adults. J Nutr Health Aging 2018, 22(9):1066-1071. 
30. Nakakubo S, Doi T, Makizako H, Tsutsumimoto K, Kurita S, Kim M, Ishii H, Suzuki T, Shimada H: Association of sleep condition and social frailty in community-dwelling older people. Geriatr Gerontol Int 2019, 19(9):885-889.

31. Nakakubo S, Doi T, Makizako H, Tsutsumimoto K, Hotta R, Kurita S, Kim M, Suzuki T, Shimada H: Sleep condition and cognitive decline in Japanese community-dwelling older people: Data from a 4year longitudinal study. J Sleep Res 2019, 28(4):e12803.

32. Shimada H, Makizako H, Doi T, Tsutsumimoto K, Lee S, Suzuki T: Cognitive Impairment and Disability in Older Japanese Adults. Plos One 2016, 11(7):e0158720.

33. Makizako H, Shimada H, Doi T, Tsutsumimoto K, Suzuki T: Impact of physical frailty on disability in community-dwelling older adults: a prospective cohort study. BMJ Open 2015, 5(9):e008462.

34. Makizako H, Shimada H, Tsutsumimoto K, Lee S, Doi T, Nakakubo S, Hotta R, Suzuki T: Social Frailty in Community-Dwelling Older Adults as a Risk Factor for Disability. Journal of the American Medical Directors Association 2015, 16(11):1003 e1007-1011.

35. Patel SR, Blackwell T, Ancoli-Israel S, Stone KL, Osteoporotic Fractures in Men-Mr OSRG: Sleep characteristics of self-reported long sleepers. Sleep 2012, 35(5):641-648.

36. Goldman SE, Stone KL, Ancoli-Israel S, Blackwell T, Ewing SK, Boudreau R, Cauley JA, Hall M, Matthews KA, Newman AB: Poor sleep is associated with poorer physical performance and greater functional limitations in older women. Sleep 2007, 30(10):1317-1324.

37. Dunlop DD, Song J, Semanik PA, Sharma L, Bathon JM, Eaton CB, Hochberg MC, Jackson RD, Kwoh CK, Mysiw WJ et al: Relation of physical activity time to incident disability in community dwelling adults with or at risk of knee arthritis: prospective cohort study. BMJ 2014, 348:g2472.

38. Fielding RA, Guralnik JM, King AC, Pahor M, McDermott MM, Tudor-Locke C, Manini TM, Glynn NW, Marsh AP, Axtell RS et al: Dose of physical activity, physical functioning and disability risk in mobility-limited older adults: Results from the LIFE study randomized trial. PLoS One 2017, 12(8):e0182155.

39. Shah RC, Buchman AS, Leurgans S, Boyle PA, Bennett DA: Association of total daily physical activity with disability in community-dwelling older persons: a prospective cohort study. BMC Geriatr 2012, 12:63.

40. Heiland EG, Welmer AK, Wang R, Santoni G, Fratiglioni L, Qiu C: Cardiovascular Risk Factors and the Risk of Disability in Older Adults: Variation by Age and Functional Status. Journal of the American Medical Directors Association 2019, 20(2):208-212 e203.

41. Choi M, Yeom HA, Jung D: Association between physical activity and metabolic syndrome in older adults in Korea: analysis of data from the Korean National Health and Nutrition Examination Survey IV. Nurs Health Sci 2013, 15(3):379-386.

42. Guedes DP, Hatmann AC, Martini FA, Borges MB, Bernardelli R, Jr.: Quality of life and physical activity in a sample of Brazilian older adults. J Aging Health 2012, 24(2):212-226.

43. Ryu M, Jo J, Lee Y, Chung YS, Kim KM, Baek WC: Association of physical activity with sarcopenia and sarcopenic obesity in community-dwelling older adults: the Fourth Korea National Health and 
Nutrition Examination Survey. Age Ageing 2013, 42(6):734-740.

44. Wermelinger Avila MP, Correa JC, Lucchetti ALG, Lucchetti G: The Role of Physical Activity in the Association Between Resilience and Mental Health in Older Adults. J Aging Phys Act 2018, 26(2):248-253.

45. Kline CE, Zielinski MR, Devlin TM, Kripke DF, Bogan RK, Youngstedt SD: Self-reported long sleep in older adults is closely related to objective time in bed. Sleep Biol Rhythms 2010, 8(1):42-51.

46. Lauderdale DS, Knutson KL, Yan LL, Liu K, Rathouz PJ: Self-reported and measured sleep duration: how similar are they? Epidemiology 2008, 19(6):838-845.

47. Tomioka K, Iwamoto J, Saeki K, Okamoto N: Reliability and validity of the International Physical Activity Questionnaire (IPAQ) in elderly adults: the Fujiwara-kyo Study. J Epidemio/ 2011, 21(6):459465.

\section{Tables}

Table 1. Characteristics of participants. 
Overall Independent $(n=4,372)$

Age (year)

Women

Education level (year)

Medications (number)

Body mass index $\left(\mathrm{kg} / \mathrm{m}^{2}\right)$

Mini Mental State

Examination (points)

Geriatric depression scale (points)

Current alcohol consumption

Never

Past

Current

Current smoking habit

Never

Past

Current

Disease (yes)

Hypertension

Diabetes

Hyperlipidemia

Respiratory disease

Long sleep duration

Physical inactivity

$75.85 \pm 4.23 \quad 75.11 \pm$

3.72

2,343

(53.6)

1,826

(52.3)

$12.02 \pm 2.59$

12.13

$\pm$

$\pm \quad 2.58$

$3.35 \pm 2.83$

3.09

$\pm \quad 2.65$

$4.38 \pm$

22.92

$\pm \quad 3.00$

22.95

$\pm$

$26.15 \pm 232$

$26.28 \pm$

2.28

$25.61 \pm 2.39$

0.001

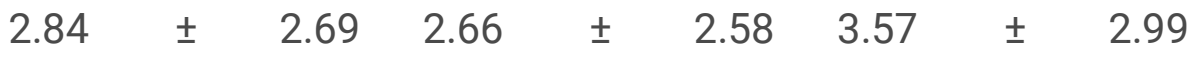

0.001

0.001

0.001

0.001

0.001

0.001

0.200 
Values are presented as $\mathrm{n}(\%)$, mean \pm SD. Continuous variables were analyzed by $t$-test, and categorical variables were analyzed by $c^{2}$ tests.

Table 2. Hazard ratios for incident disability during 5 years according to long sleep duration and physical inactivity.

\begin{tabular}{|c|c|c|c|c|c|c|c|c|c|}
\hline \multirow[b]{3}{*}{$\begin{array}{l}\text { Long sleep } \\
\text { duration }\end{array}$} & \multicolumn{3}{|c|}{ Model 1} & \multicolumn{3}{|c|}{ Model 2} & \multicolumn{3}{|c|}{ Model 3} \\
\hline & \multicolumn{2}{|c|}{$\mathrm{HR}(95 \% \mathrm{Cl})$} & \multirow{2}{*}{$\begin{array}{l}p \\
0.019\end{array}$} & \multicolumn{2}{|c|}{$\mathrm{HR}(95 \% \mathrm{Cl})$} & \multirow[t]{2}{*}{$p$} & \multicolumn{2}{|c|}{$\mathrm{HR}(95 \% \mathrm{Cl})$} & \multirow{2}{*}{$\begin{array}{l}p \\
0.026\end{array}$} \\
\hline & 1.32 & $\begin{array}{l}(1.05- \\
1.67)\end{array}$ & & & & & 1.31 & $\begin{array}{l}(1.03- \\
1.65)\end{array}$ & \\
\hline $\begin{array}{l}\text { Physical } \\
\text { inactivity }\end{array}$ & & & & 1.33 & $\begin{array}{l}(1.13- \\
1.57)\end{array}$ & $<.001$ & 1.32 & $\begin{array}{l}(1.13- \\
1.56)\end{array}$ & $<.001$ \\
\hline
\end{tabular}

HR: hazard ratio, Cl: confidence interval. All models were adjusted for age, sex, BMI, education, medication, GDS score and MMSE score, medical history, current drinking habit, current smoking habit.

Model 1 and Model 2: Long sleep duration and physical inactivity were individually submitted as independent variables.

Model 3: Long sleep duration and physical inactivity were simultaneously submitted as independent variables.

\section{Figures}




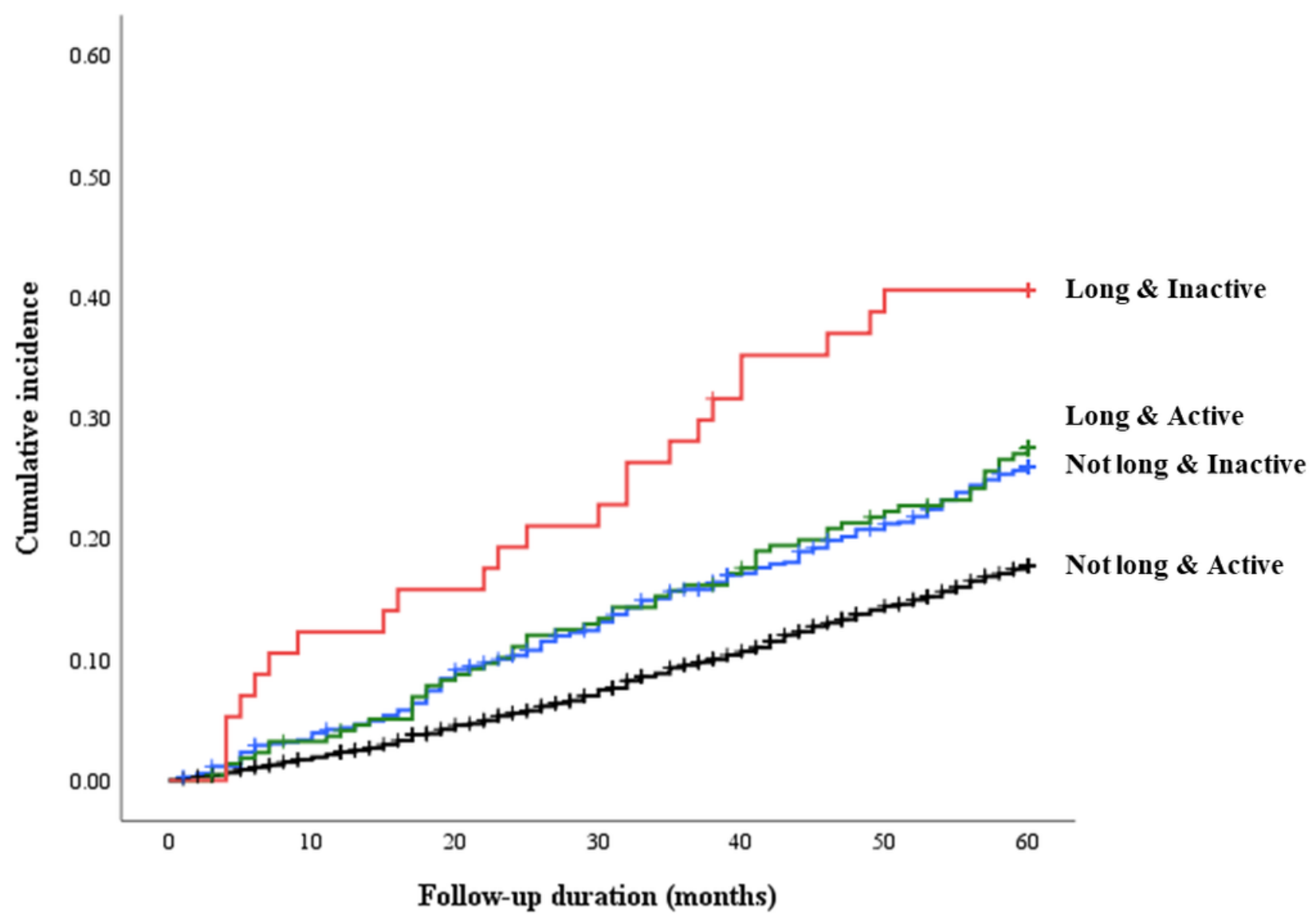

Figure 1

Kaplan-Meier survival curves of older adults showing the relationship of sleep duration and physical activity with incident disability during a 5-year follow up period.

Sleep duration \& physical activity

Not long \& Active

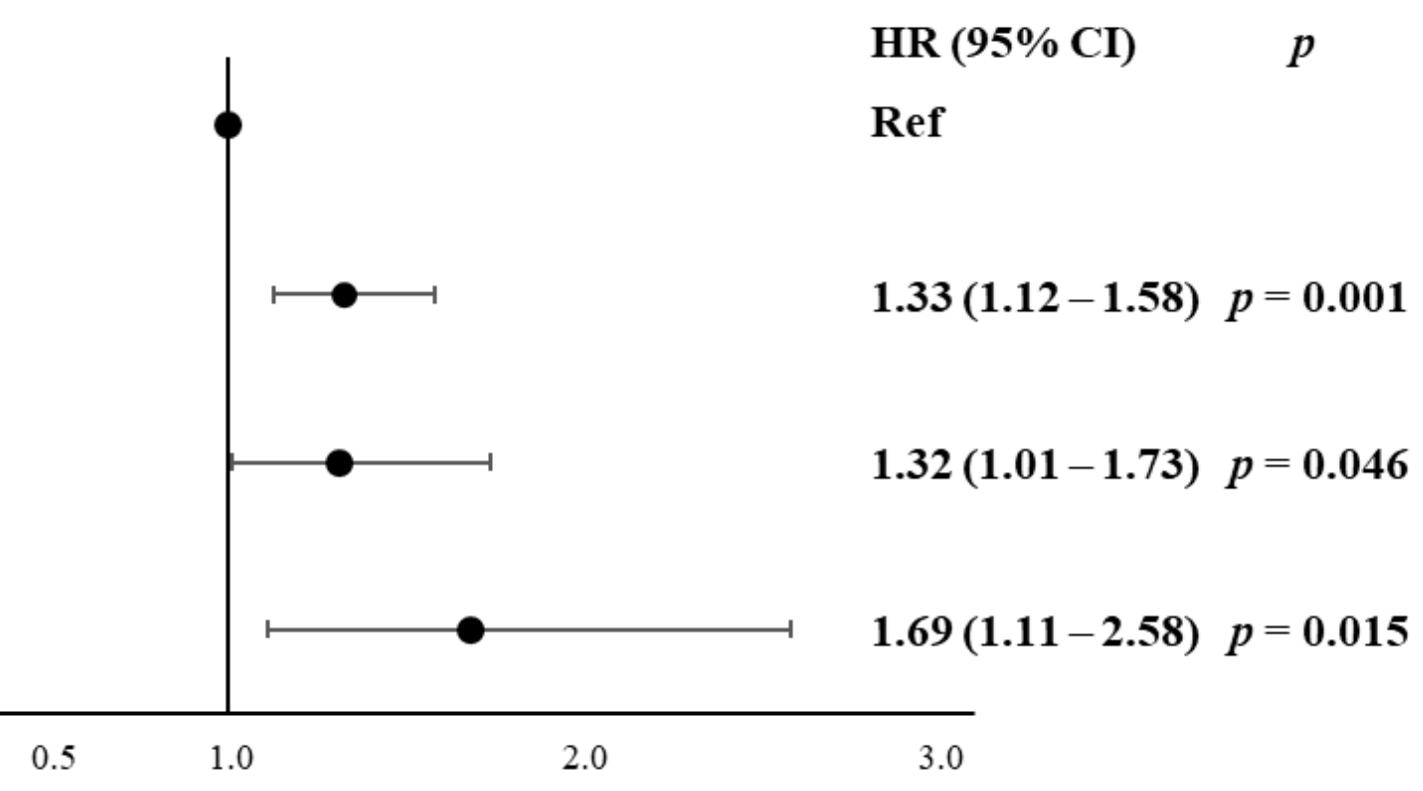




\section{Figure 2}

Multivariate Cox proportional hazard regression analysis adjusted for age, sex, BMI, education, medication, medical history, current drinking habit, current smoking habit, and GDS and MMSE scores. 